\title{
Numeri caratteristici dei flocculi d'idrogeno e di calcio c dei filamenti d'idrogeno per l'anno 1958
}

\author{
G. GoDOLI
}

Proseguendo la pubblicazione annuale dei numeri caratteristici dei flocculi d'idrogeno e di calcio e dei filamenti d'idrogeno ( $\left.{ }^{3}\right)$, vengono riportati nelle seguenti tre tabelle di immediata interpretazione $\mathrm{i}$ valori giornalieri per il 1958.

I numeri caratteristici sono stati stimati sugli spettroeliogrammi eseguiti alla torre solare di Arcetri con l'immagine di $63 \mathrm{~mm}$; le caratteristiche strumentali e la tecnica delle osservazioni sono state descritte in altra sede $\left({ }^{2}\right)$.

Durante tutto il 1958 sono stati eseguiti quotidianamente tre spettroeliogrammi successivi nella riga $H \alpha_{3}$ dell'idrogeno e tre spettroeliogrammi successivi nella riga $K_{2,3,2}$ del calcio ionizzato. L'esecuzione di spettroeliogrammi successivi ha lo scopo di mettere in evidenza le variazioni di intensità delle zone più attive. Tali variazioni di intensità, interessantissime nello studio dell'evoluzione dei fenomeni, non hanno però alcuna influenza nella stima dei numeri caratteristici. Per questo abbiamo continuato a stimare i numeri caratteristici su di un solo spettroeliogramma in $H \alpha_{3}$ ed uno solo in $K_{2.3,2}$. Gli spettroeliogrammi in $H \alpha_{3}$ esaminati erano generalmente i primi eseguiti. Solo in qualche raro caso, quando il primo risultava difettoso, veniva esaminato il secondo od il terzo spettroeliogramma. Gli spettroeliogrammi in $K_{2,3,2}$ esaminati erano quelli ridotti in sede di programma AGI.

Come è noto, l'osservatorio astrofisico di Arcetri è Centro Mondiale $\mathrm{C}$ Principale ( $\mathrm{P}$ WDC $\mathrm{C}$ ) per la raccolta ed elaborazione dei dati relativi ai flocculi di calcio $\left({ }^{3}\right)$. Per questo, dal $1^{\circ}$ gennaio 1957 l'attività dei flocculi di calcio viene seguita rilevando area e posizione dei singoli fenomeni. Sebbene questo metodo di determinare l'attività dei flocculi sia più informativo della stima del numero caratteristico e sebbene il numero caratteristico sia in media una funzione nota dell'area $\left({ }^{4}\right)$, pure abbiamo anche continuato a stimare i numeri caratteristici dei flocculi 
Tabella I - NUMERI CARATTERISTICI DEI FLOCCULI D'IDROGENO PER L'ANNO 1958

\begin{tabular}{|c|c|c|c|c|c|c|c|c|c|c|c|c|}
\hline Giorno & 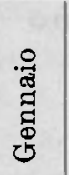 & $\begin{array}{l}0 \\
\frac{0}{3} \\
5 \\
0 \\
0 \\
0 \\
0 \\
0\end{array}$ & $\underset{\Sigma}{\stackrel{\text { N }}{\Sigma}}$ & $\stackrel{0}{=0}$ & 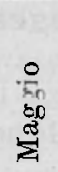 & 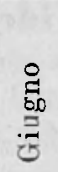 & 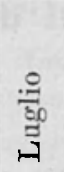 & 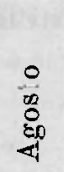 & 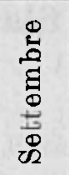 & 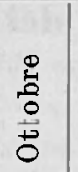 & $\begin{array}{l}0 \\
0 \\
\Xi \\
\Xi \\
8 \\
0 \\
0 \\
2\end{array}$ & 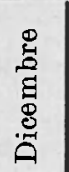 \\
\hline & 一 & 1.6 & 1.8 & 2.2 & 1.8 & - & - & 2.0 & - & - & 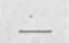 & \\
\hline 2 & 2.1 & 1.0 & - & - & 2.0 & - & 1.9 & 1.9 & 1.4 & - & $1.4^{*}$ & $2.1 *$ \\
\hline 3 & 2.0 & - & 1.4 & - & 1.9 & 2.4 & 1.8 & - & 1.5 & 1.9 & - & $2.1 *$ \\
\hline 4 & - & 1.9 & 2.0 & - & - & - & 2.1 & 1.8 & 1.5 & 2.0 & $1.6 *$ & 1.8 \\
\hline 5 & - & - & 2.0 & - & 2.2 & - & - & 2.0 & 1.6 & - & 1.5 & 1.9 \\
\hline 6 & - & 2.3 & 2.0 & - & - & - & - & 2.0 & 1.5 & $2.1 *$ & $1.5^{*}$ & 1.8 \\
\hline 7 & - & - & - & - & - & 2.2 & 2.6 & - & - & - & - & $1.9 *$ \\
\hline 8 & 2.3 & - & - & 1.8 & 1.8 & - & 2.6 & 1.6 & 1.6 & 1.9 & 0.9 & $1.8 *$ \\
\hline 9 & 2.5 & - & - & - & 1.9 & 3.2 & 2.4 & 1.6 & 1.6 & 1.8 & $0.9^{*}$ & - \\
\hline 10 & 2.6 & - & - & 1.7 & 1.9 & - & 2.3 & - & - & & - & \\
\hline 11 & - & - & - & - & - & — & 2.3 & 2.2 & 1.7 & 1.8 & - & 2.2 \\
\hline 12 & - & 2.3 & - & 一 & 1.8 & - & 2.0 & 1.9 & 1.8 & - & - & 2.2 \\
\hline 13 & - & 2.3 & - & - & 2.0 & - & - & 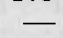 & 1.6 & - & - & - \\
\hline 14 & - & - & - & 1.4 & 1.9 & - & 1.9 & 2.0 & - & 1.7 & $0.9^{*}$ & - \\
\hline 15 & 2.5 & - & 1.3 & - & - & - & 1.9 & - & 1.8 & 1.9 & 0.9 & — \\
\hline 16 & 2.3 & - & - & - & - & 1.6 & 1.9 & 2.1 & 1.9 & - & $1.0 *$ & $1.6 *$ \\
\hline 17 & 2.6 & - & - & — & - & - & 1.9 & - & 2.5 & 1.8 & 1.0 & - \\
\hline 18 & 2.2 & - & - & - & - & - & 1.9 & 1.8 & 2.3 & 1.7 & 1.0 & $1.9 *$ \\
\hline 19 & - & - & - & 1.8 & 1.8 & - & 1.9 & 1.8 & 2.2 & - & 1.0 & $2.0 *$ \\
\hline 20 & - & 1.8 & - & - & 1.9 & - & - & 1.8 & 2.1 & - & 1.2 & - \\
\hline 21 & 1.6 & 1.9 & - & 2.2 & 2.0 & - & 1.8 & - & - & 1.8 & $1.4^{*}$ & - \\
\hline 22 & 1.8 & - & 1.0 & - & - & - & - & 1.9 & 2.2 & 2.0 & $1.8 *$ & 2.1 \\
\hline 23 & 1.9 & - & - & - & - & - & - & 1.8 & 1.8 & $1.8 *$ & - & \\
\hline 24 & 2.2 & - & - & 2.6 & 2.1 & - & - & - & 2.0 & 1.7 & $2.2^{*}$ & $2.0 *$ \\
\hline 25 & 2.3 & - & - & 2.4 & - & 2.0 & 2.0 & 2.0 & 1.9 & 1.7 & - & $2.3 *$ \\
\hline 26 & - & - & - & 2.2 & 2.2 & - & 2.1 & 2.4 & 2.1 & $1.4^{*}$ & - & 2.2 \\
\hline 27 & 1.5 & 1.9 & - & - & 2.2 & - & - & 2.3 & - & 1.3 & $2.8^{*}$ & $2.0^{*}$ \\
\hline 28 & 1.4 & - & - & 2.2 & 2.1 & - & 2.0 & 2.1 & - & 1.4 & $2.6 *$ & - \\
\hline 0 & 1.5 & - & - & 1.8 & 2.2 & - & - & 2.0 & 2.0 & 1.6 & 2.0 & - \\
\hline 30 & 1.4 & - & - & 2.0 & & - & 2.2 & - & - & $1.6^{*}$ & $2.3^{*}$ & 2.0 \\
\hline 31 & 1.5 & - & - & - & 2.3 & - & 2.1 & - & - & $1.6 *$ & & \\
\hline Media & 2.0 & 2.0 & 1.6 & 2.0 & 2.0 & 2.3 & 2.1 & 2.0 & 1.8 & 1.7 & 1.5 & 2.0 \\
\hline $\begin{array}{c}\text { Media } \\
\text { semestrale }\end{array}$ & \multicolumn{6}{|c|}{1.99} & \multicolumn{6}{|c|}{1.85} \\
\hline $\begin{array}{l}\text { Media } \\
\text { annuale }\end{array}$ & \multicolumn{12}{|c|}{.9} \\
\hline
\end{tabular}


NUMERI CARATTERISTICI DEI FLOCCULI D'IDROGENO E DI CALCIO, ECC. 199

Tabella 2 - Numeri CaRatteristici Dei flocculi di Calcio PER L'ANNO 1958

\begin{tabular}{|c|c|c|c|c|c|c|c|c|c|c|c|c|}
\hline Giorno & 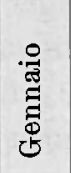 & $\begin{array}{l}7 \\
\frac{7}{3} \\
0 \\
0 \\
0 \\
0 \\
0\end{array}$ & 离 & 苋 & 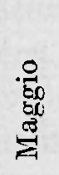 & 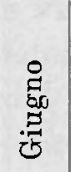 & 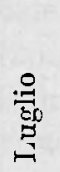 & 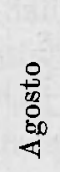 & 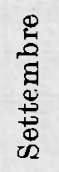 & \begin{tabular}{l}
0 \\
\multirow{2}{0}{} \\
0 \\
0 \\
+ \\
0 \\
0
\end{tabular} & $\begin{array}{l}0 \\
\text { है } \\
\text { घี } \\
8 \\
0 \\
\text { ¿ }\end{array}$ & 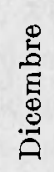 \\
\hline 1 & - & 2.2 & 2.7 & - & 2.9 & -1 & - & 4.3 & & - & - & - \\
\hline 2 & 2.9 & & - & - & 3.0 & 一 & 3.0 & 4.2 & 3.2 & - & - & - \\
\hline 3 & - & - & 2.3 & - & 3.1 & 3.3 & 3.2 & - & 3.1 & 2.7 & - & - \\
\hline 4 & - & - & 2.5 & - & & 3.2 & 3.1 & 3.8 & 3.2 & 2.8 & - & 3.5 \\
\hline 5 & -1 & - & 2.5 & - & 2.8 & - & - & 3.9 & 3.3 & - & 2.6 & 3.5 \\
\hline 6 & -1 & 3.2 & 2.4 & - & - & -1 & - & 4.0 & 3.1 & - & - & 3.4 \\
\hline 7 & - & - & - & - & - & 3.3 & 3.6 & - & - & - & - & - \\
\hline 8 & 3.1 & - & - & 2.8 & 2.8 & - & 3.5 & 3.7 & - & 2.6 & 2.6 & - \\
\hline 9 & 3.1 & - & - & - & 2.9 & 3.1 & 3.5 & 3.5 & 3.2 & 2.7 & - & - \\
\hline 10 & 3.3 & - & 一 & 3.2 & 2.6 & - & 3.5 & - & - & - & - & - \\
\hline 11 & - & - & - & - & - & - & 3.4 & 3.5 & 3.4 & 2.5 & - & - \\
\hline 12 & - & 3.2 & -1 & - & 2.7 & - & 3.0 & 3.4 & - & - & - & 3.5 \\
\hline 13 & - & 3.1 & -1 & - & 2.8 & - & & & 3.3 & - & - & - \\
\hline 14 & - & - & - & 2.2 & 2.6 & - & 2.9 & 3.0 & - & 2.9 & - & 一 \\
\hline 15 & - & - & - & - & - & - & 3.0 & - & 3.3 & 2.8 & 2.5 & - \\
\hline 16 & 3.1 & - & - & - & - & 2.5 & 3.1 & 3.1 & 3.3 & - & - & - \\
\hline 17 & 2.9 & - & - & - & - & - & - & - & - & 2.8 & 2.6 & - \\
\hline 18 & 3.0 & - & - & - & - & - & 3.2 & 2.9 & 3.1 & 2.8 & 3.0 & - \\
\hline 19 & - & - & - & 2.5 & 2.3 & - & 3.2 & 2.8 & 3.2 & - & 3.0 & - \\
\hline 20 & - & 2.4 & - & - & 2.1 & - & - & 3.0 & 3.0 & - & 3.0 & - \\
\hline 21 & - & - & - & 2.6 & 2.3 & -1 & 3.2 & - & - & 2.7 & - & - \\
\hline 22 & 2.9 & - & 2.3 & - & - & -1 & - & - & 2.9 & 2.7 & - & - \\
\hline 23 & 2.8 & - & - & - & - & - & - & 3.4 & 3.0 & - & - & - \\
\hline 24 & 2.6 & - & - & 2.7 & 2.8 & - & - & - & 3.2 & 2.4 & - & - \\
\hline 25 & 2.6 & - & - & 3.2 & & 3.3 & 3.2 & 3.7 & 3.1 & 2.3 & - & - \\
\hline 26 & - & - & - & 3.1 & 3.3 & - & 3.4 & 3.8 & 3.0 & - & - & - \\
\hline 27 & 2.5 & 2.1 & - & - & 3.2 & -1 & - & 3.8 & - & 2.1 & - & - \\
\hline 28 & 2.4 & - & - & 3.4 & 3.3 & - & 3.8 & 3.8 & - & 2.2 & - & - \\
\hline 29 & 2.4 & - & - & 3.4 & 3.2 & -1 & - & 3.4 & 2.9 & 2.5 & 3.6 & - \\
\hline $\begin{array}{l}30 \\
31\end{array}$ & 2.5 & Z & - & 3.2 & $\overline{3.1}$ & 二 & $\begin{array}{l}4.3 \\
4.3\end{array}$ & 二 & 2.8 & 2.2 & E & 二 \\
\hline Media & 2.8 & 2.7 & 2.5 & 2.9 & 2.8 & 3.1 & 3.4 & 3.6 & 3.1 & 2.6 & 2.9 & 3.5 \\
\hline $\begin{array}{l}\text { Media } \\
\text { semestrale }\end{array}$ & \multicolumn{6}{|c|}{2.82} & \multicolumn{6}{|c|}{3.16} \\
\hline $\begin{array}{c}\text { Modia } \\
\text { annuale }\end{array}$ & \multicolumn{12}{|c|}{3.02} \\
\hline
\end{tabular}


Tabella 3 - Numeri CARATteristici DeI FILAMENTI D'IDRogeno PER L'ANNO 1958

\begin{tabular}{|c|c|c|c|c|c|c|c|c|c|c|c|c|}
\hline Giorno & $\begin{array}{c}\stackrel{\varrho}{\Xi} \\
\stackrel{\Xi}{\Xi} \\
0 \\
\stackrel{\Xi}{\Xi}\end{array}$ & 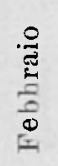 & $\stackrel{尺}{\stackrel{2}{E}}$ & 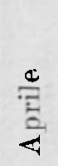 & 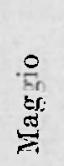 & 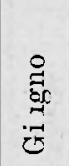 & $\frac{0}{\frac{0}{g}}$ & $\begin{array}{l}0 \\
\stackrel{0}{n} \\
\stackrel{0}{8} \\
\stackrel{8}{8}\end{array}$ & 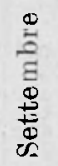 & $\begin{array}{l}0 \\
\stackrel{0}{2} \\
0 \\
0 \\
0 \\
0\end{array}$ & 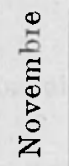 & 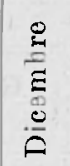 \\
\hline 1 & & 1.6 & 2.3 & 2.2 & 2.3 & - & - & 1.8 & & - & 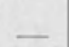 & - \\
\hline 2 & 2.8 & - & - & - & 2.0 & - & 2.4 & 1.7 & 2.3 & - & $1.6 *$ & $2.3^{*}$ \\
\hline 3 & 2.7 & - & 2.5 & - & 1.4 & 2.0 & 2.3 & - & 2.4 & 2.0 & - & $2.3^{*}$ \\
\hline 4 & 一 & 2.2 & 3.2 & - & - & - & 2.2 & 1.8 & 2.4 & 2.2 & $2.3 *$ & 2.4 \\
\hline 5 & - & - & 3.0 & - & 1.9 & - & - & 1.9 & 2.4 & - & 1.8 & 2.3 \\
\hline 6 & - & 2.4 & 2.8 & - & - & - & - & 2.2 & 2.4 & $2.5^{*}$ & $2.2 *$ & 2.2 \\
\hline 7 & - & - & - & - & - & 1.4 & 2.2 & - & - & - & - & $2.5^{*}$ \\
\hline 8 & 2.6 & - & - & 2.3 & 2.2 & - & 2.1 & 2.6 & 2.2 & 2.3 & 1.6 & $2.5^{*}$ \\
\hline 9 & 2.7 & - & - & - & 2.7 & 1.6 & 2.3 & 2.3 & 2.4 & 2.2 & $2.4^{*}$ & - \\
\hline 10 & 2.8 & - & - & 2.4 & 2.8 & - & 2.1 & - & & & - & - \\
\hline 11 & - & - & - & - & - & - & 2.2 & 2.5 & 2.4 & 2.3 & - & 2.4 \\
\hline 12 & - & 3.1 & - & - & 2.3 & - & 2.3 & 2.4 & 2.0 & - & - & 2.6 \\
\hline 13 & - & 2.9 & - & - & 1.9 & - & . & - & 2.1 & - & - & - \\
\hline 14 & - & - & - & 2.8 & 2.0 & - & 1.1 & 2.2 & - & 2.2 & $2.0 *$ & - \\
\hline 15 & 2.4 & - & 2.4 & - & - & - & 1.4 & - & 1.8 & 2.3 & 1.7 & - \\
\hline 16 & 2.4 & - & - & - & - & 2.4 & 1.5 & 2.2 & 1.7 & - & $2.0^{*}$ & $1.9^{*}$ \\
\hline 17 & 2.5 & - & - & - & - & - & 1.7 & - & 1.8 & 1.2 & 1.9 & - \\
\hline 18 & 2.4 & - & - & - & - & - & 2.2 & 1.9 & 2.3 & 1.1 & 2.2 & $2.6^{*}$ \\
\hline 19 & - & - & - & 2.5 & 1.9 & - & 2.2 & 2.0 & 2.2 & - & 2.1 & $2.9 *$ \\
\hline 20 & $\overline{0}$ & 2.3 & - & - & 1.4 & - & - & 2.3 & 2.5 & - & 2.3 & - \\
\hline 21 & 2.1 & 2.4 & - & 2.6 & 2.4 & - & 2.6 & - & - & 1.2 & $2.6^{*}$ & - \\
\hline 22 & 2.8 & - & 1.0 & - & - & - & - & 2.4 & 3.2 & 1.4 & $2.5 *$ & 2.3 \\
\hline 23 & 2.6 & - & - & - & - & - & - & 2.6 & 3.1 & $1.8^{*}$ & - & - \\
\hline 24 & 2.2 & - & - & 2.6 & 2.8 & -- & - & - & 2.9 & 1.4 & $2.0^{*}$ & $2.4^{*}$ \\
\hline 25 & 2.3 & - & - & 2.5 & - & 2.3 & 2.7 & 2.5 & 2.6 & 1.4 & - & $2.5^{*}$ \\
\hline 26 & & - & - & 2.4 & 3.0 & - & 2.8 & 2.5 & 2.7 & $1.4^{*}$ & - & $2.6^{*}$ \\
\hline 27 & 2.3 & 2.5 & - & - & 2.7 & - & - & 2.8 & - & 1.2 & $2.0^{*}$ & $2.5^{*}$ \\
\hline 28 & 2.4 & - & - & 2.4 & 2.5 & - & 1.8 & 3.0 & - & 1.3 & $2.0 *$ & - \\
\hline 29 & 2.5 & - & - & 2.4 & 2.2 & - & - & 2.5 & 2.0 & 1.4 & 2.0 & - \\
\hline 30 & 2.6 & - & - & 2.4 & - & - & 1.9 & - & - & $1.5^{*}$ & $2.0^{*}$ & 2.6 \\
\hline 31 & 1.9 & - & - & - & 2.0 & - & 1.7 & - & - & $1.6^{*}$ & - & \\
\hline Media & 2.5 & 2.4 & 2.5 & 2.5 & 2.2 & 1.9 & 2.1 & 2.3 & 2.4 & 1.7 & 2.1 & 2.4 \\
\hline $\begin{array}{c}\text { Media } \\
\text { semestrale }\end{array}$ & \multicolumn{6}{|c|}{2.36} & & \multicolumn{5}{|c|}{2.15} \\
\hline $\begin{array}{l}\text { Media } \\
\text { annuale }\end{array}$ & \multicolumn{12}{|c|}{2.23} \\
\hline
\end{tabular}


di calcio: sembra infatti che in fasi peculiari del ciclo solare, i due diversi metodi diano informazioni di significato diverso $\left({ }^{5}\right)$.

Dall'ottobre 1958 è stato iniziato ad Arcetri un programma di osservazione fotografica sistematica al monocromatore Halle in $H \alpha$ che già era in funzione per l'osservazione visuale dall'agosto $1957\left({ }^{8}\right)$. Vengono giornalmente eseguiti diversi fotogrammi formato $24 \times 36 \mathrm{~mm}$ ad intervalli di 10-15 minuti. Se vengono osservati visualmente fenomeni a rapida evoluzione quali brillamenti o protuberanze eruttive, la frequenza dei fotogrammi viene aumentata adeguandola alla variabilità del fenomeno. L'osservazione fotografica al monocromatore è molto più rapida che allo spettroeliografo cosicchè in molti giorni misti in cui non è possibile eseguire spettroeliogrammi alla torre solare, è invece possibile effettuare filtrogrammi al monocromatore. Un filtrogramma per ognuno di tali giorni è stato ingrandito a $63 \mathrm{~mm}$ di diametro ed esaminato per la determinazione dei numeri caratteristici dei flocculi e filamenti in $H \alpha_{3}$. I numeri caratteristici cosi dedotti sono individuati in tabella 1 con un asterisco.

Sono stati esaminati complessivamente 165 spettroeliogrammi e 28 filtrogrammi in $H \alpha_{3}$ e 152 spettroeliogrammi in $K_{2,3,2}$.

La stima veniva eseguita, come per il passato, attenendosi ai modelli distribuiti dall'osservatorio di Mt. Wilson. Per evidenti ragioni di omogeneità e costanza di giudizio, è stata determinata prima tutta la serie dei numeri caratteristici per i flocculi d'idrogeno; quindi tutta la serie per i flocculi di calcio ed infine tutta la serie per i filamenti d'idrogeno.

Le stime sono state eseguite per due volte seguendo un diverso ordine di esame. Lo scarto delle singole determinazioni si è sempre mantenuto inferiore a 0.3 unità. Nei pochi casi in cui veniva riscontrato uno scarto di maggior entità, un riesame degli spettroeliogrammi metteva in evidenza errori di stima.

Il contributo dei singoli osservatori alla raccolta delle lastre ridotte è stato il seguente:

\begin{tabular}{|c|r|r|r|}
\hline Osservatori & $H a_{3}$ & $K_{2,3,2}$ & Totale \\
\cline { 1 - 2 } & & & \\
M. C. Ballario . . & 110 & 104 & 214 \\
G. Godoli . . . . & 4 & 4 & 8 \\
B. Romagnoli . . & 51 & 44 & 95 \\
\hline
\end{tabular}


Il contributo dei singoli osservatori alla raccolta dei fotogrammi ridotti è stato invece il seguente:

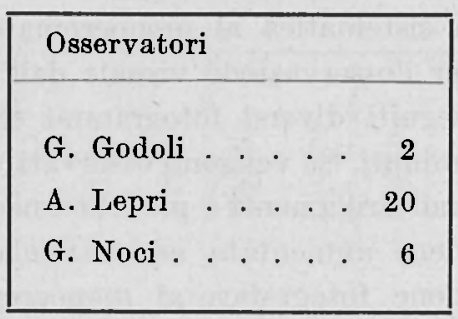

La distribuzione mensile degli spettroeliogrammi e filtrogrammi ridotti è la seguente:

\begin{tabular}{|c|c|c|c|c|c|c|c|c|c|c|c|c|}
\hline$\lambda$ mese & 1 & 2 & 3 & 4 & 5 & 6 & 7 & 8 & 9 & 10 & 11 & 12 \\
\hline s. e.g. & 19 & 8 & 7 & 12 & 19 & 5 & 21 & 21 & 22 & 16 & 8 & 7 \\
\hline fot. & - & - & - & - & - & - & - & - & - & 5 & 12 & 10 \\
\hline$K_{2,3,2}$ & 15 & 6 & 6 & 11 & 19 & 6 & 20 & 20 & 20 & 17 & 8 & 4 \\
\hline
\end{tabular}

\section{RIASSUNTO}

Si danno $i$ numeri caratteristici dei flocculi d'idrogeno e di calcio e dei filamenti d'idrogeno per l'anno 1958, secondo le osservazioni eseguite alla torre solare ed al monocromatore Halle dell'osservatorio di Arcetri.

\section{$A B S T R A C T$}

The character figures of $\mathrm{Ca}$ bright flocculi and $\mathrm{H \alpha}$ bright and dark flocculi for the 1958 are given according to the observations carried out at the solar tower and $H \alpha$ filter of the Arcetri observatory. 
NUMERI CARATTERISTICI DEI FTOCCULI D'IDROGENO E DI CALCIO, ECC. 203

\section{BIBLIOGRAFIA}

(1) Godoli, G., Numeri caratteristici dei flocculi d'idrogeno e di calcio e dei filamenti d'idrogeno per gli anni 1950-51-52, "Ann. Geof. ", 6, 199 (1953).

- Idem per l'anno 1953, 1. c. 7, 135, (1954).

- Idem per l'anno 1954, l. c. 8, 325, (1955).

- Idem per l'anno 1955, 1. c. 9, 299, (1956).

- Idem per l'anno 1956, 1. c. 10, 133, (1957).

- Idem per l'anno 1957, 1. c. 11, 235, (1957).

(2) Righini, G. - Godoli, G., Riduzione del materiale spettroeliografico raccolto alla torre solare di Arcetri nel periodo 1932.1949, "Mem. SAIt", 21, 333 (1949).

$\left({ }^{3}\right)$ Godoli, G., Relazione preliminare sull'attività del " Principal World Data Center $A G I$ " ad Arcetri, "La ricerca scientifica ", in corso di stampa.

(4) Righini, G. - Godoli, G., Sui numeri caratteristici dell'attività solare, "Ann. Geof.", 3, 501, (1950).

- - The physical meaning of the character figures of solar phenomena, "J. of Geophys. Res. ", 55, 415, (1950).

(5) Godoli, G., Attività dei flocculi d'idrogeno e di calcio e dei flamenti d'idrogeno per il periodo 1o luglio 1957-30 giugno 1958 "Boll. di Geod. e Scienze Affini ", 17, 487, (1958).

$\left({ }^{6}\right)$ Rigutti, M., Il filtro interferenziale dell'osservatorio astrofisico di Arcetri, "La ricerca scientifica", in corso di stampa. 